\title{
Quantification of quantum steering in a Gaussian Greenberger-Horne-Zeilinger state
}

\author{
Xiaowei Deng ${ }^{1}$, Caixing Tian $^{1}$, Meihong Wang ${ }^{1}$, Zhongzhong Qin ${ }^{1,2}$, and Xiaolong Su ${ }^{1,2 *}$ \\ ${ }^{1}$ State Key Laboratory of Quantum Optics and Quantum Optics Devices, \\ Institute of Opto-Electronics, Shanxi University, \\ Taiyuan, 030006, People's Republic of China \\ ${ }^{2}$ Collaborative Innovation Center of Extreme Optics, \\ Shanxi University, Taiyuan 030006, China
}

\begin{abstract}
As one of the most intriguing features of quantum mechanics, Einstein-Podolsky-Rosen (EPR) steering is a useful resource for secure quantum networks. Greenberger-Horne-Zeilinger (GHZ) state plays important role in quantum communication network. By reconstructing the covariance matrix of a continuous variable tripartite GHZ state, we fully quantify the amount of bipartite steering under Gaussian measurements. We demonstrate that the (1+1)-mode steerability is not exist in the tripartite GHZ state, only the collectively steerability exist between the (1+2)-mode and (2+1)-mode partitions. These properties confirm that the tripartite GHZ state is a perfect resource for quantum secret sharing protocol. We also demonstrate one-way EPR steering of the GHZ state under Gaussian measurements, and experimentally verify the introduced monogamy relations for Gaussian steerability. Our experiment provides reference for using EPR steering in Gaussian GHZ states as a valuable resource for multiparty quantum information tasks.
\end{abstract}

\section{INTRODUCTION}

Einstein-Podolsky-Rosen (EPR) steering, the phenomenon that one party, Alice, can steer the state of a distant party, Bob, by local measurements on Alice is an intriguing phenomenon predicted by quantum mechanics [1-4]. In the hierarchy of quantum correlations, EPR steering represents a weaker form of quantum nonlocality and stands between Bell nonlocality [5, 6] and EPR entanglement [7]. Such correlation is intrinsically asymmetric between the two subsystems [8-18], which allows verification of EPR steering when one subsystem is untrusted [19], while the verification of entanglement is on the premise that trusted devices are used [7, 20], and the Bell nonlocality premises no need of trustiness between each other. Based on this asymmetric feature, EPR steering is known as a potential resource for one-sided device-independent (1sDI) quantum key distribution [21-25], secure quantum teleportation [26-28], and subchannel discrimination [29].

The one-way EPR steering with a two-mode squeezed state [16] and genuine one-way EPR steering have been experimentally demonstrated [17, 18]. It has been experimentally demonstrated that the direction of one-way EPR steering can be actively manipulated [30], which may lead to more consideration in the application of EPR steering. There are also some theoretical analysis about EPR steering among multipartite quantum state generated in different physical systems [31-34]. Experimental observation of multipartite EPR steering has been reported in optical network [35, 36] and photonic qubits [37, 38].

The theoretical study of monogamy relations [32, 39-42] offers insights into understanding whether and how this special type of quantum correlation can be distributed over many different systems. In a pure three-mode Gaussian states, the residual Gaussian steering from a monogamy inequality [32]

*Electronic address: suxl@sxu.edu.cn

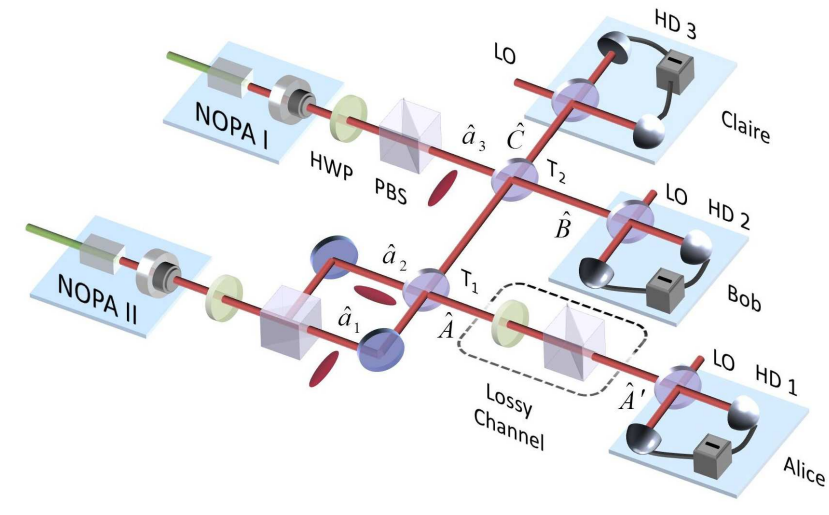

FIG. 1: The experimental set-up. The squeezed states $\left(\hat{a}_{1}, \hat{a}_{2}\right.$ and $\hat{a}_{3}$ ) with $-2.94 \mathrm{~dB}$ squeezing at the sideband frequency of $3 \mathrm{MHz}$ are generated from two nondegenerate optical parametric amplifiers (NOPAs). $T_{1}$ and $T_{2}$ are the beam-splitters used to generate the tripartite GHZ state. An optical mode $(\hat{A})$ of the state is distributed over a lossy quantum channel which is composed by a half-wave plate (HWP) and a polarization beam-splitter (PBS). $\mathrm{HD}_{1-3}$, homodyne detectors. LO, the local oscillator.

has been demonstrated that can be used to quantify the genuine multipartite steering [31] and acquires an operational interpretation in the context of a 1sDI quantum secret sharing protocol [43]. Very recently, the monogamy relations for EPR steering in Gaussian cluster state has been demonstrated experimentally [36].

Greenberger-Horne-Zeilinger (GHZ) state, a kind of multipartite entangled states, is an important resource for constructing quantum network [44]. For example, it has been used in quantum teleportation network [45], controlled dense coding [46], and quantum secret sharing [43, 47]. Recently, the quantum entanglement swapping between two Gaussian GHZ state has also been demonstrated [48].

In this paper, the distribution of quantum steering in a con- 
tinuous variable (CV) tripartite GHZ state of optical field and the monogamy relation [32, 40] of quantum steering are theoretically and experimentally studied. With the reconstructed covariance matrix of the GHZ state obtained from the measurement results, the EPR steering in various bipartite splits under Gaussian measurements are quantified. We find that a given mode of the GHZ state cannot be steered by another mode of the state, this property is different from those of a CV four-mode cluster state [36]. However, the collective steerabilities between $(1+2)$-mode and $(2+1)$-mode partitions are observed. We further precisely validate the monogamy relations proposed for Gaussian steering in the presence of loss [32, 40, 41]. This study clearly reveals the distribution of quantum steering among different parties in tripartite Gaussian GHZ states. Also, these characteristics demonstrate that the CV tripartite GHZ state is a perfect resource of quantum secret sharing protocol [43].

The paper is organized as follows. We present the preparation of the tripartite GHZ state in Sec. II. The details of the experiment are presented in Sec. III. The results and discussion are presented in Sec. IV. Finally, we conclude the paper in Sec. V.

\section{PREPARATION OF THE TRIPARTITE GHZ STATE}

The quantum state used in the experiment is a CV tripartite GHZ entangled state [44-46]. The tripartite entangled state is prepared deterministically by coupling a phase-squeezed state $\left(\hat{a}_{2}\right)$ of light and two amplitude-squeezed states of light $\left(\hat{a}_{1}\right.$ and $\left.\hat{a}_{3}\right)$ on an optical beam-splitter network, which consists of two optical beam-splitters with transmittance of $T_{1}=1 / 3$ and $T_{2}=1 / 2$, respectively, as shown in Fig. 1. Three input squeezed states are expressed by

$$
\begin{aligned}
& \hat{a}_{1}=\frac{1}{2}\left[e^{-r_{1}} \hat{x}_{1}^{(0)}+i e^{r_{1}} \hat{p}_{1}^{(0)}\right], \\
& \hat{a}_{2}=\frac{1}{2}\left[e^{r_{2}} \hat{x}_{2}^{(0)}+i e^{-r_{2}} \hat{p}_{2}^{(0)}\right], \\
& \hat{a}_{3}=\frac{1}{2}\left[e^{-r_{3}} \hat{x}_{3}^{(0)}+i e^{r_{3}} \hat{p}_{3}^{(0)}\right],
\end{aligned}
$$

where $r_{i}(i=1,2,3)$ is the squeezing parameter, $\hat{x}=\hat{a}+\hat{a}^{\dagger}$ and $\hat{p}=\left(\hat{a}-\hat{a}^{\dagger}\right) / i$ are the amplitude and phase quadratures of an optical field $\hat{a}$, respectively, and the superscript of the amplitude and phase quadratures represent the vacuum state. The transformation matrix of the beam-splitter network is given by

$$
U=\left[\begin{array}{ccc}
\sqrt{\frac{2}{3}} & \sqrt{\frac{1}{3}} & 0 \\
-\sqrt{\frac{1}{6}} & \sqrt{\frac{1}{3}} & \sqrt{\frac{1}{2}} \\
-\sqrt{\frac{1}{6}} & \sqrt{\frac{1}{3}} & -\sqrt{\frac{1}{2}}
\end{array}\right],
$$

the unitary matrix can be decomposed into a beam-splitter network $U=B_{23}\left(T_{2}\right) I_{2}(-1) B_{12}\left(T_{1}\right)$, where $B_{k l}\left(T_{j}\right)$ stands for the linearly optical transformation on $j$-th beam-splitter with transmission of $T_{j}(j=1,2)$, where $\left(B_{k l}\right)_{k k}=$ $\sqrt{1-T},\left(B_{k l}\right)_{k l}=\left(B_{k l}\right)_{l k}=\sqrt{T},\left(B_{k l}\right)_{l l}=-\sqrt{1-T}$, are matrix elements of the beam-splitter. $I_{k}(-1)=e^{i \pi}$ corresponds to a $180^{\circ}$ rotation in phase space. The output modes from the optical beam-splitter network are

$$
\begin{aligned}
& \hat{A}=\sqrt{\frac{2}{3}} \hat{a}_{1}+\sqrt{\frac{1}{3}} \hat{a}_{2}, \\
& \hat{B}=-\sqrt{\frac{1}{6}} \hat{a}_{1}+\sqrt{\frac{1}{3}} \hat{a}_{2}+\sqrt{\frac{1}{2}} \hat{a}_{3}, \\
& \hat{C}=-\sqrt{\frac{1}{6}} \hat{a}_{1}+\sqrt{\frac{1}{3}} \hat{a}_{2}-\sqrt{\frac{1}{2}} \hat{a}_{3},
\end{aligned}
$$

respectively. Here, we have assumed that three squeezed states have the identical squeezing parameter $\left(r_{1}=r_{2}=\right.$ $r_{3}=r$ ). In experiments, the requirement is achieved by adjusting the two nondegenerate optical parametric amplifiers (NOPAs) to operate at same conditions. For our experimental system, we have $r=0.339$, which corresponding to -2.94 $\mathrm{dB}$ squeezing. The correlation variances between the amplitude and phase quadratures of the tripartite entangled state are expressed by $\Delta^{2}\left(\hat{x}_{A}-\hat{x}_{B}\right)=\Delta^{2}\left(\hat{x}_{A}-\hat{x}_{C}\right)=\Delta^{2}\left(\hat{x}_{B}-\hat{x}_{C}\right)=$ $2 e^{-2 r}$ and $\Delta^{2}\left(\hat{p}_{A}+\hat{p}_{B}+\hat{p}_{C}\right)=3 e^{-2 r}$, respectively.

In the experiment, the optical mode $\hat{A}$ of the state is distributed in a lossy channel, which is mimicked by a beamsplitter composed by a half-wave plate and a polarization beam-splitter (Fig. 1). The output mode is given by $\hat{A}^{\prime}=$ $\sqrt{\eta} \hat{A}+\sqrt{1-\eta} \hat{v}$, where $\eta$ and $\hat{v}$ represent the transmission efficiency of the quantum channel and the vacuum mode induced by loss into the quantum channel, respectively. The bipartite steerabilities among modes $\hat{A}^{\prime}, \hat{B}$ and $\hat{C}$ are investigated.

The properties of a $\left(n_{A}+m_{B}\right)$-mode Gaussian state $\rho_{A B}$ of a bipartite system can be determined by its covariance matrix

$$
\sigma_{A B}=\left(\begin{array}{cc}
A & C \\
C^{\top} & B
\end{array}\right)
$$

with elements $\sigma_{i j}=\left\langle\hat{\xi}_{i} \hat{\xi}_{j}+\hat{\xi}_{j} \hat{\xi}_{i}\right\rangle / 2-\left\langle\hat{\xi}_{i}\right\rangle\left\langle\hat{\xi}_{j}\right\rangle$, where $\hat{\xi} \equiv$ $\left(\hat{x}_{1}^{A}, \hat{p}_{1}^{A}, \ldots, \hat{x}_{n}^{A}, \hat{p}_{n}^{A}, \hat{x}_{1}^{B}, \hat{p}_{1}^{B}, \ldots, \hat{x}_{m}^{B}, \hat{p}_{m}^{B}\right)$ is the vector of the amplitude and phase quadratures of optical modes. The submatrices $A$ and $B$ are corresponding to the reduced states of Alice's and Bob's subsystems, respectively.

Based on the covariance matrix of the state, the steerability of Bob by Alice $(A \rightarrow B)$ for a $\left(n_{A}+m_{B}\right)$-mode Gaussian state can be quantified by [14]

$$
\mathcal{G}^{A \rightarrow B}\left(\sigma_{A B}\right)=\max \left\{0,-\sum_{j: \bar{v}_{j}^{A B \backslash A}<1} \ln \left(\bar{v}_{j}^{A B \backslash A}\right)\right\},
$$

where $\bar{v}_{j}^{A B \backslash A}\left(j=1, \ldots, m_{B}\right)$ are the symplectic eigenvalues of $\bar{\sigma}_{A B \backslash A}=B-C^{\top} A^{-1} C$, derived from the Schur complement of $A$ in the covariance matrix $\sigma_{A B}$. The quantity $G^{A \rightarrow B}$ is a monotone under Gaussian local operations and classical communication [41] and vanishes iff the state described by $\sigma_{A B}$ is nonsteerable by Gaussian measurements [14]. The steerability of Alice by Bob $\left[G^{B \rightarrow A}\left(\sigma_{A B}\right)\right]$ can be obtained by swapping the roles of $A$ and $B$. 

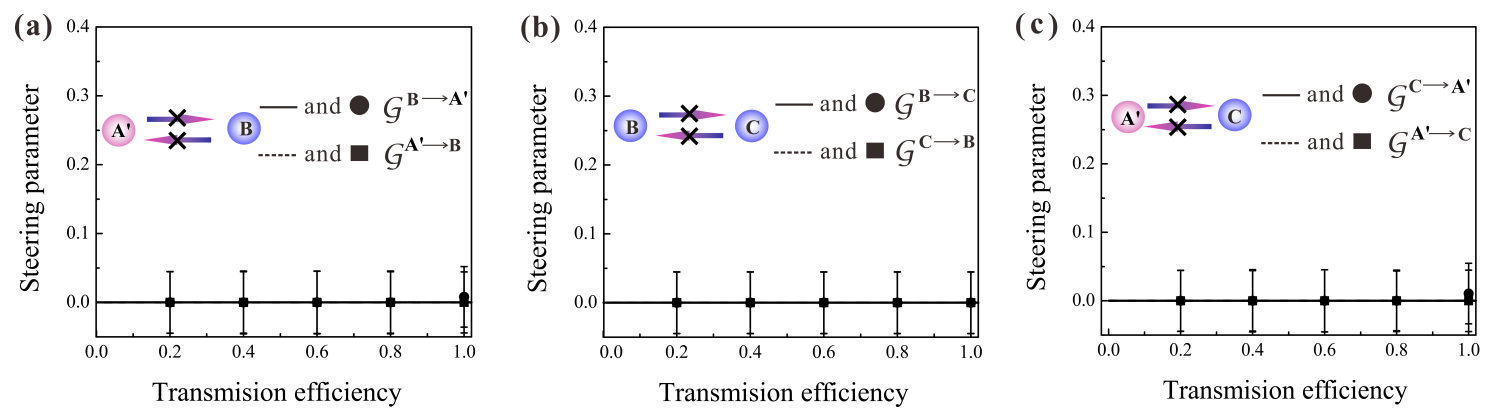

FIG. 2: EPR steering between any two modes of the GHZ state. (a - c) There are no EPR steering between modes $\hat{A}^{\prime}$ and $\hat{B}, \hat{B}$ and $\hat{C}, \hat{A}^{\prime}$ and $\hat{C}$, respectively, under Gaussian measurements. In all the panels, the quantities plotted are dimensionless. The dots and squares represent the experimental data measured at different transmission efficiencies. Error bars represent \pm one standard deviation and are obtained based on the statistics of the measured covariance matrices.

\section{DETAILS OF THE EXPERIMENT}

In the experiment, the $\hat{x}$-squeezed and $\hat{p}$-squeezed states are produced by non-degenerate optical parametric amplifiers (NOPAs) pumped by a common laser source, which is a continuous wave intracavity frequency-doubled and frequencystabilized Nd:YAP-LBO (Nd-doped $\mathrm{YAlO}_{3}$ perorskite-lithium triborate) laser. The fundamental wave at $1080 \mathrm{~nm}$ wavelength is used for the injected signals of NOPAs and the local oscillators of homodyne detectors. The second-harmonic wave at $540 \mathrm{~nm}$ wavelength serves as the pump field of the NOPAs, in which through an intracavity frequency-downconversion process a pair of signal and idler modes with the identical frequency at $1080 \mathrm{~nm}$ and the orthogonal polarizations are generated.

Each of NOPAs consists of an $\alpha$-cut type-II KTiOPO4 (KTP) crystal and a concave mirror. The front face of KTP crystal is coated to be used for the input coupler and the concave mirror serves as the output coupler of squeezed states, which is mounted on a piezo-electric transducer for locking actively the cavity length of NOPAs on resonance with the injected signal at $1080 \mathrm{~nm}$. The transmissivities of the front face of KTP crystal at $540 \mathrm{~nm}$ and $1080 \mathrm{~nm}$ are $21.2 \%$ and $0.04 \%$, respectively. The end-face of KTP is cut to $1^{\circ}$ along $\mathrm{y}-\mathrm{z}$ plane of the crystal and is antireflection coated for both $1080 \mathrm{~nm}$ and $540 \mathrm{~nm}$ [49]. The transmissivities of output coupler at $540 \mathrm{~nm}$ and $1080 \mathrm{~nm}$ are $0.5 \%$ and $12.5 \%$, respectively. In our experiment, all NOPAs are operated at the parametric deamplification situation [49, 50]. Under this condition, the coupled modes at $+45^{\circ}$ and $-45^{\circ}$ polarization directions are the $\hat{x}$-squeezed and $\hat{p}$-squeezed states, respectively [50]. The quantum efficiency of the photodiodes used in the homodyne detectors are $95 \%$. The interference efficiency on all beamsplitters are about $99 \%$.

In our experiment, the partially reconstructed covariance matrix $\sigma_{A^{\prime} B C}$, which corresponds to the distributed mode $\hat{A}^{\prime}$ and modes $\hat{B}, \hat{C}$, is measured by three homodyne detec- tors [51]. The covariance matrix $\sigma$ is given by

$$
\sigma=\left[\begin{array}{ccc}
\sigma_{A^{\prime}} & \sigma_{A^{\prime} B} & \sigma_{A^{\prime} C} \\
\sigma_{A^{\prime} B}^{T} & \sigma_{B} & \sigma_{B C} \\
\sigma_{A^{\prime} C}^{T} & \sigma_{B C}^{T} & \sigma_{C}
\end{array}\right],
$$

Thus, the three-mode covariance matrix can be partially expressed as (the cross correlations between different quadratures of one mode are taken as 0 )

$$
\begin{aligned}
\sigma_{A} & =\left[\begin{array}{cc}
\Delta^{2} \hat{x}_{A^{\prime}} & 0 \\
0 & \Delta^{2} \hat{p}_{A^{\prime}}
\end{array}\right], \\
\sigma_{B} & =\left[\begin{array}{cc}
\Delta^{2} \hat{x}_{B} & 0 \\
0 & \Delta^{2} \hat{p}_{B}
\end{array}\right], \\
\sigma_{C} & =\left[\begin{array}{cc}
\Delta^{2} \hat{x}_{C} & 0 \\
0 & \Delta^{2} \hat{p}_{C}
\end{array}\right], \\
\sigma_{A B} & =\left[\begin{array}{cc}
\operatorname{Cov}\left(\hat{x}_{A^{\prime}}, \hat{x}_{B}\right) & \operatorname{Cov}\left(\hat{x}_{A^{\prime}}, \hat{p}_{B}\right) \\
\operatorname{Cov}\left(\hat{p}_{A^{\prime}}, \hat{x}_{B}\right) & \operatorname{Cov}\left(\hat{p}_{A^{\prime}}, \hat{p}_{B}\right)
\end{array}\right], \\
\sigma_{A C} & =\left[\begin{array}{ll}
\operatorname{Cov}\left(\hat{x}_{A^{\prime}}, \hat{x}_{C}\right) & \operatorname{Cov}\left(\hat{x}_{A^{\prime}}, \hat{p}_{C}\right) \\
\operatorname{Cov}\left(\hat{p}_{A^{\prime}}, \hat{x}_{C}\right) & \operatorname{Cov}\left(\hat{p}_{A^{\prime}}, \hat{p}_{C}\right)
\end{array}\right], \\
\sigma_{B C} & =\left[\begin{array}{ll}
\operatorname{Cov}\left(\hat{x}_{B}, \hat{x}_{C}\right) & \operatorname{Cov}\left(\hat{x}_{B}, \hat{p}_{C}\right) \\
\operatorname{Cov}\left(\hat{p}_{B}, \hat{x}_{C}\right) & \operatorname{Cov}\left(\hat{p}_{B}, \hat{p}_{C}\right)
\end{array}\right] .
\end{aligned}
$$

where $\operatorname{Cov}(\hat{x}, \hat{p})$ is the covariance between two corresponding quadratures. To partially reconstruct all relevant entries of the associated covariance matrix of the state, we perform 18 different measurements on the output optical modes. These measurements include the amplitude and phase quadratures of the output optical modes, and the cross correlations $\Delta^{2}\left(\hat{x}_{A^{\prime}}-\hat{x}_{B}\right), \Delta^{2}\left(\hat{x}_{A^{\prime}}-\hat{x}_{C}\right), \Delta^{2}\left(\hat{x}_{B}-\hat{x}_{C}\right), \Delta^{2}\left(\hat{p}_{A^{\prime}}-\hat{p}_{B}\right)$, $\Delta^{2}\left(\hat{p}_{A^{\prime}}-\hat{p}_{C}\right), \quad \Delta^{2}\left(\hat{p}_{B}-\hat{p}_{C}\right), \Delta^{2}\left(\hat{x}_{A^{\prime}}+\hat{p}_{B}\right), \Delta^{2}\left(\hat{x}_{A^{\prime}}+\hat{p}_{C}\right)$, $\Delta^{2}\left(\hat{x}_{B}+\hat{p}_{C}\right), \Delta^{2}\left(\hat{p}_{A^{\prime}}+\hat{x}_{B}\right), \Delta^{2}\left(\hat{p}_{A^{\prime}}+\hat{x}_{C}\right)$ and $\Delta^{2}\left(\hat{p}_{B}+\hat{x}_{C}\right)$. The covariance elements are calculated via the identities [51]

$$
\begin{aligned}
& \operatorname{Cov}\left(\hat{\xi}_{i}, \hat{\xi}_{j}\right)=\frac{1}{2}\left[\Delta^{2}\left(\hat{\xi}_{i}+\hat{\xi}_{j}\right)-\Delta^{2} \hat{\xi}_{i}-\Delta^{2} \hat{\xi}_{j}\right], \\
& \operatorname{Cov}\left(\hat{\xi}_{i}, \hat{\xi}_{j}\right)=-\frac{1}{2}\left[\Delta^{2}\left(\hat{\xi}_{i}-\hat{\xi}_{j}\right)-\Delta^{2} \hat{\xi}_{i}-\Delta^{2} \hat{\xi}_{j}\right] .
\end{aligned}
$$

In the experiment, we measured three covariance matrices for each quantum state and got the mean steering parameters from the corresponding mean values. 

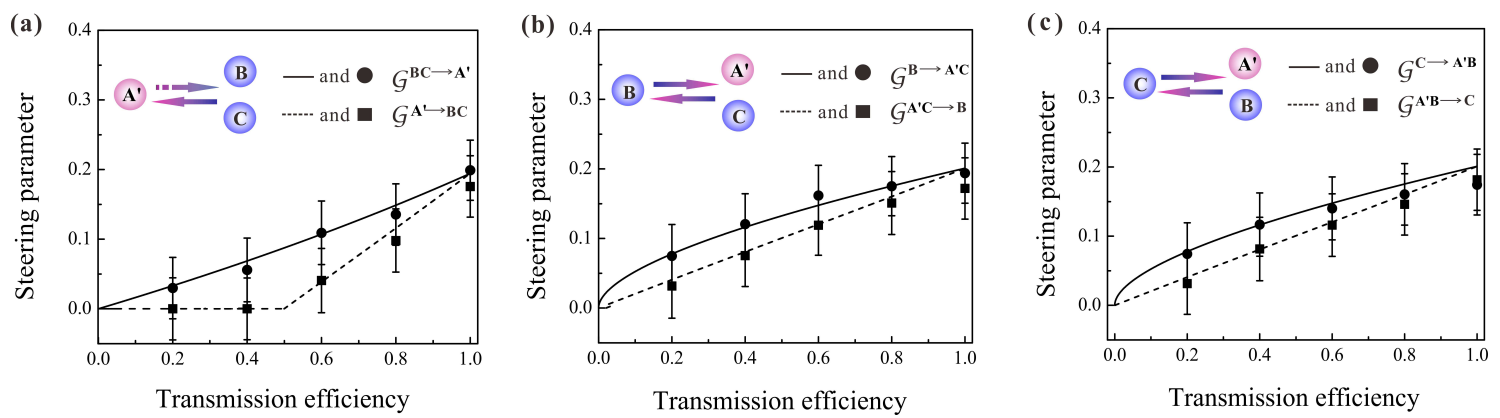

FIG. 3: EPR steering between one and two modes of the GHZ state. (b) One-way EPR steering between modes $\hat{A}^{\prime}$ and $\{\hat{B}, \hat{C}\}$ under Gaussian measurements. (b) Modes $\hat{B}$ and $\left\{\hat{A}^{\prime}, \hat{C}\right\}$ can steer each other asymmetrically and the steerability grows with increasing transmission efficiency. (c) Modes $\hat{C}$ and $\left\{\hat{A}^{\prime}, \hat{B}\right\}$ can steer each other asymmetrically and the steerability grows with increasing transmission efficiency. In all the panels, the quantities plotted are dimensionless. The dots and squares represent the experimental data measured at different transmission efficiencies. Error bars represent \pm one standard deviation and are obtained based on the statistics of the measured covariance matrices.
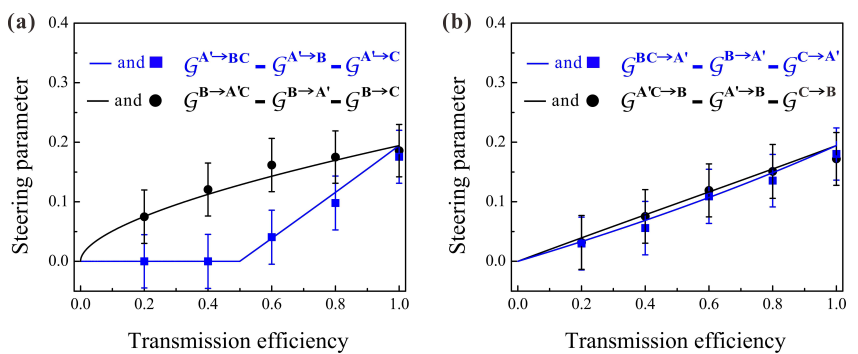

FIG. 4: Demonstration of monogamy relations. (a) Monogamy relation of steering quantifier for $(1+2)$-mode partitions. (b) Monogamy relation of steering quantifier for $(2+1)$-mode partitions. In both the panels, the quantities plotted are dimensionless. The dots and squares represent the experimental data measured at different transmission efficiencies. Error bars represent \pm one standard deviation and are obtained based on the statistics of the measured covariance matrices.

\section{RESULTS AND DISCUSSIONS}

The experimental results of the quantum steerability between any two modes $[(1+1)$-mode $]$ of the tripartite GHZ state under Gaussian measurements are shown in Figure 2. We demonstrate that there is no steering exist between any two modes. We can account for the observation by the monogamy relation as shown in ref. [40]: one mode cannot be steered by two distinct modes simultaneously. In fact, the GHZ state is fully symmetric under mode permutations [39, 47], i.e., modes $\hat{A}, \hat{B}$ and $\hat{C}$ are totally symmetric. Thus, if $\hat{A}^{\prime}$ could be steered by $\hat{B}$, it should be equally steered by $\hat{C}$, which is forbidden by the monogamy relation [40].

While, the scenario of the steering in a CV four-mode square Gaussian cluster state shown in Ref. [36] shows different result. There is no steering between any two neighboring modes of the four-mode square cluster state, since the two neighbors of one mode are symmetric and the steering is forbidden by the monogamy relation [40]. And the twomode steering presents between diagonal modes because in that structure any mode has just one unique diagonal mode, thus it suffers no constraint from the monogamy relation [40].

Figure 3 shows the steerability between one mode and the other two modes of the GHZ state, i.e., $(1+2)$-mode and $(2+1)$-mode partitions. Interestingly, we stress that any two modes $\{\hat{\imath}, \hat{\jmath}\}$ can collectively steer another mode $\hat{k}$. We further measure the steerability when the steered party comprises two modes. Inequal steerability between $\mathcal{G}^{B C \rightarrow A^{\prime}}$ and $\mathcal{G}^{A^{\prime} \rightarrow B C}$ under Gaussian measurements is shown due to the loss imposed on $\hat{A}$, and one-way steering is observed during $\eta \in(0,0.5]$, as shown in Fig. 3(a). As shown in Fig. 3(b), modes $\left\{\hat{A}^{\prime}, \hat{C}\right\}$ and mode $\hat{B}$ can always steer each other. The same result is observed for the steerability between modes $\left\{\hat{A}^{\prime}, \hat{B}\right\}$ and $\hat{C}$, as shown in Fig. 3(c).

Since quantum secret sharing can be implemented when the two players are separated in a local quantum network and collaborate to decode the secret sent by the dealer who own the other one mode [43]. From the results in Fig. 2 and Fig. 3 , we demonstrate that steerability doesn't exist between any two subsystems, but the collectively steerability exist between the $(1+2)$-mode and $(2+1)$-mode partitions. These properties confirm that the $\mathrm{CV}$ tripartite GHZ state is a perfect resource of quantum secret sharing protocol.

In addition, our results $\mathcal{G}^{B \rightarrow A^{\prime} C}>0$ [Fig. 3(b)], $\mathcal{G}^{C \rightarrow A^{\prime} B}>0$ [Fig. 3(c)] and $\mathcal{G}^{A^{\prime} \rightarrow B C}>0$ when $\eta>0.5$ [Fig. 3(a)] also confirm experimentally that when the steered system is composed of at least two modes, the monogamy relation shown in ref. [32] is demonstrated, such that the system can be steered by more than one party simultaneously [32]. With these results, we present the experimental demonstration of the monogamy relation, Coffman-Kundu-Wootters (CKW)-type monogamy which is seminal studied in entanglement [39], quantifies the distribution of the steering among different subsystems [32]. For a state contains three-mode, the CKW-type monogamy relation presents

$$
\begin{aligned}
& \mathcal{G}^{k \rightarrow(i, j)}\left(\sigma_{i j k}\right)-\mathcal{G}^{k \rightarrow i}\left(\sigma_{i j k}\right)-\mathcal{G}^{k \rightarrow j}\left(\sigma_{i j k}\right) \geq 0, \\
& \mathcal{G}^{(i, j) \rightarrow k}\left(\sigma_{i j k}\right)-\mathcal{G}^{i \rightarrow k}\left(\sigma_{i j k}\right)-\mathcal{G}^{j \rightarrow k}\left(\sigma_{i j k}\right) \geq 0,
\end{aligned}
$$

where $i, j, k \in\left\{\hat{A}^{\prime}, \hat{B}, \hat{C}\right\}$. All possible configurational types of 
$(1+2)$-mode and $(2+1)$-mode steering monogamy relation has been experimentally verified with our experimental results, as shown in Fig. 4.

\section{CONCLUSION}

In summary, we fully quantify the steering characterization for all bipartite configurations with the deterministically generated GHZ state and the reconstructed covariance matrix under Gaussian measurements. The distribution of EPR steering over (1+1)-mode, $(1+2)$-mode and (2+1)-mode partitions of a CV tripartite Gaussian GHZ state subject to asymmetric loss have been investigated. The fact that a given mode of the state cannot be steered by another mode is observed, and the collective steerabilities between $(1+2)$-mode and $(2+1)$ mode partitions are observed. These results demonstrate that the CV tripartite GHZ state is a perfect resource of quantum secret sharing protocol.
We also provide experimental confirmation for two types of monogamy relations for the CV tripartite Gaussian GHZ state, which bound the distribution of steerability among different modes. Our work thus provides a concrete in-depth understanding of EPR steering in paradigmatic multipartite states such as GHZ states, and advances our fundamental knowledge of monogamy relations for Gaussian steerability. This work thus can be useful in establishing secure teleportation fidelity thresholds, and bounds on 1sDI quantum key distribution and secret sharing among many sites over lossy quantum channels.

Acknowledgments This research was supported by National Natural Science Foundation of China (Grants No. 11522433 and No. 61475092), the program of Youth Sanjin Scholar, National Basic Research Program of China (Grant No. 2016YFA0301402), and the Fund for Shanxi "1331 Project" Key Subjects Construction.
[1] E. Schrödinger, Discussion of probability relations between separated systems, Proc. Cambridge Philos. Soc. 31 (1935) 555-563.

[2] M.D. Reid, Demonstration of the Einstein-Podolsky-Rosen paradox using nondegenerate parametric amplification, Phys. Rev. A 40 (1989) 913-923.

[3] H.M. Wiseman, S.J. Jones, A.C. Doherty, Steering, entanglement, nonlocality, and the Einstein-Podolsky-Rosen paradox Phys. Rev. Lett. 98 (14) (2007) 140402.

[4] D. Cavalcant, P. Skrzypczyk, Quantum steering: a review with focus on semidefinite programming, Rep. Prog. Phys. 80 (2017) 024001 .

[5] J.S. Bell, On the Einstein Podolsky Rosen paradox, Physics 1 (1964) 195-200.

[6] N. Brunner, D. Cavalcanti, S. Pironio, V. Scarani, S. Wehner, Bell nonlocality, Rev. Mod. Phys. 86 (2) (2014) 419-478.

[7] R. Horodecki, P. Horodecki, M. Horodecki, K. Horodecki, Quantum entanglement, Rev. Mod. Phys. 81 (2) (2009) 865942.

[8] S.L.W. Midgley, A.J. Ferris, M.K. Olsen, Asymmetric Gaussian steering: When Alice and Bob disagree, Phys. Rev. A 81 (2) (2010) 022101.

[9] S.P. Walborn, A. Salles, R.M. Gomes, F. Toscano, P.H. Souto Ribeiro, Revealing hidden Einstein-Podolsky-Rosen nonlocality, Phys. Rev. Lett. 106 (13) (2011) 130402.

[10] J. Schneeloch, C.J. Broadbent, S.P. Walborn, E.G. Cavalcanti, J.C. Howell, Einstein-Podolsky-Rosen steering inequalities from entropic uncertainty relations, Phys. Rev. A 87 (6) (2013) 062103.

[11] J. Bowles, T. Vertesi, M.T. Quintino, N. Brunner, One-way Einstein-Podolsky-Rosen steering, Phys. Rev. Lett. 112 (20) (2014) 200402.

[12] B. Opanchuk, L. Arnaud, M.D. Reid, Detecting faked continuous-variable entanglement using one-sided deviceindependent entanglement witnesses, Phys. Rev. A 89 (6) (2014) 062101.

[13] Q.Y. He, Q.H. Gong, M.D. Reid, Classifying directional Gaussian entanglement, Einstein-Podolsky-Rosen steering, and dis- cord, Phys. Rev. Lett. 114 (6) (2015) 060402.

[14] I. Kogias, A.R. Lee, S. Ragy, G. Adesso, Quantification of Gaussian quantum steering, Phys. Rev. Lett. 114 (6) (2015) 060403.

[15] L. Rosales-Zárate, R.Y. Teh, S. Kiesewetter, A. Brolis, K. Ng, M.D. Reid, Decoherence of Einstein-Podolsky-Rosen steering, J. Opt. Soc. Am. B 32 (4) (2015) A82-A91.

[16] V. Händchen, T. Eberle, S. Steinlechner, A. Samblowski, T. Franz, R.F. Werner, R. Schnabel, Observation of one-way Einstein-Podolsky-Rosen steering, Nat. Photon. 6 (2012) 596599.

[17] S. Wollmann, N. Walk, A.J. Bennet, H.M. Wiseman, G.J. Pryde, Observation of genuine one-way Einstein-PodolskyRosen steering, Phys. Rev. Lett. 116 (16) (2016) 160403.

[18] K. Sun, X.J. Ye, J.S. Xu, X.Y. Xu, J.S. Tang, Y.C. Wu, J.L. Chen, C.F. Li, G.C. Guo, Experimental quantification of asymmetric Einstein-Podolsky-Rosen steering, Phys. Rev. Lett. 116 (16) (2016) 160404.

[19] E.G. Cavalcanti, S.J. Jones, H.M. Wiseman, M.D. Reid, Experimental criteria for steering and the Einstein-Podolsky-Rosen paradox, Phys. Rev. A 80 (3) (2009) 032112.

[20] E. Schrödinger, Die gegenwärtige Situation in der Quantenmechanik, Die Naturwissenschaften 23 (1935) 823-828.

[21] M. Tomamichel, R. Renner, Uncertainty relation for smooth entropies, Phys. Rev. Lett. 106 (11) (2011) 110506.

[22] C. Branciard, E.G. Cavalcanti, S.P. Walborn, V. Scarani, H.M. Wiseman, One-sided device-independent quantum key distribution: security, feasibility, and the connection with steering, Phys. Rev. A 85 (1) (2012) 010301(R).

[23] N. Walk, S. Hosseini, J. Geng, O. Thearle, J.Y. Haw, S. Armstrong, S.M. Assad, J. Janousek, T.C. Ralph, T. Symul, H.M. Wiseman, P.K. Lam, Experimental demonstration of Gaussian protocols for one-sided device-independent quantum key distribution, Optica 3 (6) (2016) 634-642.

[24] T. Gehring, V. Händchen, J. Duhme, F. Furrer, T. Franz, C. Pacher, R.F. Werner, R. Schnabel, Implementation of continuous-variable quantum key distribution with composable and one-sided-device independent security against coherent at- 
tacks, Nat. Commun. 6 (2015) 8795.

[25] R. Gallego, L. Aolita, Resource theory of steering, Phys. Rev. X 5 (4) (2015) 041008.

[26] M.D. Reid, Signifying quantum benchmarks for qubit teleportation and secure quantum communication using EinsteinPodolsky-Rosen steering inequalities, Phys. Rev. A 88 (6) (2013) 062338.

[27] Q.Y. He, L. Rosales-Zárate, G. Adesso, M.D. Reid, Secure continuous variable teleportation and Einstein-Podolsky-Rosen steering, Phys. Rev. Lett. 115 (18) (2015) 180502.

[28] C.Y. Chiu, N. Lambert, T.L. Liao, F. Nori, C.M. Li, Nocloning of quantum steering, NPJ Quantum Information 2 (2016) 16020.

[29] M. Piani, J. Watrous, Necessary and sufficient quantum information characterization of Einstein-Podolsky-Rosen steering, Phys. Rev. Lett. 114 (6) (2015) 060404.

[30] Z.Z. Qin, X.W. Deng, C.X. Tian, M.H. Wang, X.L. Su, C.D. Xie, K.C. Peng, Manipulating the direction of EinsteinPodolsky-Rosen steering, Phys. Rev. A 95 (5) (2017) 052114.

[31] Q.Y. He, M.D. Reid, Genuine multipartite Einstein-PodolskyRosen steering, Phys. Rev. Lett. 111 (25) (2013) 250403.

[32] Y. Xiang, I. Kogias, G. Adesso, Q.Y He, Multipartite Gaussian steering: monogamy constraints and quantum cryptography applications, Phys. Rev. A 95 (1) (2017) 010101(R).

[33] L. Wang, S.C. Lv, J.T. Jing, Quantum steering in cascaded fourwave mixing Processes, Opt. Express 25 (2017) 017457.

[34] L. Wang, H.L. Wang, S.J. Li, Y.X. Wang, J.T. Jing, Phasesensitive cascaded four-wave-mixing processes for generating three quantum correlated beams, Phys. Rev. A 95 (1) (2017) 013811 .

[35] S. Armstrong, M. Wang, R.Y. Teh, Q.H. Gong, Q.Y. He, J. Janousek, H.A. Bachor, M.D. Reid, P.K. Lam, Multipartite Einstein-Podolsky-Rosen steering and genuine tripartite entanglement with optical networks, Nat. Phys. 11 (2015) 167172.

[36] X.W. Deng, Y. Xiang, C.X. Tian, G. Adesso, Q.Y. He, Q.H. Gong, X.L. Su, C.D. Xie, K.C. Peng, Demonstration of Monogamy Relations for Einstein-Podolsky-Rosen Steering in Gaussian Cluster States, Phys. Rev. Lett. 118 (23) (2017) 230501.

[37] D. Cavalcanti, P. Skrzypczyk, G.H. Aguilar, R.V. Nery, P.H.S. Ribeiro, S.P. Walborn, Detection of entanglement in asymmetric quantum networks and multipartite quantum steering, Nat. Commun. 6 (2015) 7941.

[38] C.M. Li, K. Chen, Y.N. Chen, Q. Zhang, Y.A. Chen, .J.W. Pan,
Genuine high-order Einstein-Podolsky-Rosen steering, Phys. Rev. Lett. 115 (1) (2015) 010402.

[39] V. Coffman, K. J, W.K. Wootters, Distributed entanglement, Phys. Rev. A 61 (5) (2013) 052306.

[40] M.D. Reid, Monogamy inequalities for the Einstein-PodolskyRosen paradox and quantum steering, Phys. Rev. A 88 (6) (2013) 062108.

[41] Lami L, Hirche C, Adesso G and Winter A 2016 Schur complement inequalities for covariance matrices and monogamy of quantum correlations Phys. Rev. Lett. 117220502

[42] S. Cheng, A. Milne, M.J.W. Halland, H.M. Wiseman, Volume monogamy of quantum steering ellipsoids for multiqubit systems, Phys. Rev. A 94 (4) (2016) 042105.

[43] I. Kogias, Y. Xiang, Q.Y. He, G. Adesso, Unconditional security of entanglement-based continuous variable quantum secret sharing, Phys. Rev. A 95 (1) (2017) 012315.

[44] P. van Loock, S.L. Braunstein, Multipartite entanglement for continuous variables: a quantum teleportation network, Phys. Rev. Lett. 84 (15) (2000) 3482-3485.

[45] H. Yonezawa, T. Aoki, A. Furusawa, Demonstration of a quantum teleportation network for continuous variables, Nature 431 (2004) 430-433.

[46] J.T. Jing, J. Zhang, Y. Yan, F.G. Zhao, C.D. Xie, and K.C. Peng, Experimental demonstration of tripartite entanglement and controlled dense coding for continuous variables, Phys. Rev. Lett. 90 (16) (2003) 167903.

[47] M. Wang, Y. Xiang, Q.Y. He, Q.H. Gong 2015 Detection of quantum steering in multipartite continuous-variable Greenberger-Horne-Zeilinger-like states Phys. Rev. A 91 (1) (2015) 012112.

[48] X. Su, C. Tian, X. Deng, Q. Li, C. Xie, K. Peng, Quantum entanglement swapping between two multipartite entangled states, Phys. Rev. Lett. 117 (2016) 240503.

[49] Y. Zhou, X. Jia, F. Li, C. Xie, K. Peng, Experimental generation of $8.4 \mathrm{~dB}$ entangled state with an optical cavity involving a wedged type-II nonlinear crystal, Opt. Express 23 (2015) 49524959.

[50] X.L. Su, A.H. Tan, X.J. Jia, J. Zhang, C.D. Xie, K.C. Peng, Experimental preparation of quadripartite cluster and Greenberger-Horne-Zeilinger entangled states for continuous variables, Phys. Rev. Lett. 98 (7) (2007) 070502.

[51] S. Steinlechner, J. Bauchrowitz, T. Eberle, R. Schnabel, Strong Einstein-Podolsky-Rosen steering with unconditional entangled states, Phys. Rev. A 87 (2) (2013) 022104. 